\title{
Caractérisation phéno-morphologique de quelques lignées de fève (Vicia faba L.) sélectionnées et adaptées aux conditions de culture dans les régions arides en Tunisie
}

\author{
Bouthaina Al Mohandes Dridi (1), Mohamed Loumerem (2), \\ Samira Ibn Maaouia Houimli (1), Naouel Jabbes (1), \\ Samir Tlahig (1)
}

(I) Institut Supérieur Agronomique de Chott-Mariem, Tunisie

(2) Institut des Régions Arides, Tunisie

\begin{abstract}
Cette étude avait pour objectif la caractérisation phéno-morphologique de $\mathrm{I}_{3}$ lignées de fève sélectionnées à l'Institut des Régions Arides de Medenine (Tunisie) à partir de 42 populations traditionnelles. La variabilité phéno-morphologique de ces lignées a été documentée et suivie du semis jusqu'à la maturité des graines par des mesures biométriques et des évaluations qualitatives sur différentes parties des plantes. Les caractères morphologiques ont été décrits en se basant sur le descripteur de l'UPOV (2000) pour l'espèce Vicia faba L. L'analyse des résultats permet de distinguer 2 groupes en se basant sur le critère précocité et le poids de cent graines. Le premier groupe est constitué par la lignée 8 et se distingue par le fait qu'elle est la plus précoce et présente le poids de cent graines le plus élevé. Le deuxième groupe se subdivise en 3 sous-groupes qui diffèrent entre eux par la vigueur et la productivité de la plante. Le premier sous-groupe renferme les lignées 5, IO, II, I2 et I3 se caractérise par un nombre de fleurs par racème plus faible, avec un poids moyen de cent graines variant de I 62,96 à $165,24 \mathrm{~g}$ et des nombres de graines par gousse les plus élevés. Le deuxième sous-groupe englobe les lignées 6,7 , et 9 qui sont les plus homogènes du point de vue de la date de $50 \%$ floraison, hauteur finale de la plante et longueur de la foliole. Le dernier sous-groupe inclus les lignées I, 2, 3 et 4 qui sont caractérisées par un nombre de gousses par plante, un nombre de tiges par plante, et un nombre de fleurs par racème les plus élevés. Ces lignées devraient être conservées et valorisées pour les programmes d'amélioration variétale de l'espèce Vicia faba par des sélections successives jusqu'à l'obtention d'une variété synthétique plus productive et mieux adaptée aux conditions arides du Sud Tunisien.
\end{abstract}

Mots clés : Phénologie, agromorphologie, caractérisation, agrobiodiversité, descripteur agronomique

The objective of the present study was to produce a pheno-morphological characterization of $\mathrm{I} 3$ faba bean lines selected by the Institute of Arid Regions from 42 local populations. The pheno- 
morphological variability of these lineages was observed and followed from planting through to seed maturity by biometric measurements and by qualitative evaluations on different plant parts. Morphological characteristics were described by using the UPOV (2000) descriptor. The analysis of results made it possible to distinguish between 2 groups based on precocity and hundred-seed weight. The first group was represented by line 8 which differs from others by the fact that it was the earliest and had the highest one hundred-seed weight. The second group was divided into 3 subgroups that differed in terms of plant vigour and productivity. The first subgroup was characterized by a lower number of flowers per raceme, a hundred-seed weight average ranging from $\mathrm{I} 62.96 \mathrm{~g}$ to $\mathrm{I} 65.24 \mathrm{~g}$ and a highest number of seeds per pod. This first subgroup contained the lines 5, IO, II, I2 and I3. The second subgroup included lines 6, 7 and 9 which were the most homogenous on $50 \%$ flowering date, plant height and length of leaflet. The latter subgroup included the lines I, 2, 3 and 4, characterized by a highest number of pods per plant, number of stems per plant, and number of flowers per raceme. These lines should be preserved and valued for the varietal improvement programs of Vicia faba. Successive selection should be practiced until a more productive synthetic variety is obtained that is better adapted to the dry conditions of South Tunisia.

Key words: Phenology, agromorphology, characterization, Vicia faba

\section{Introduction}

La fève (Vicia faba L.) est l'un des légumes les plus anciennement cultivés dans le monde (Tanno \& Willcox, 2006). Cette légumineuse constitue une source de protéines importante pour l'alimentation de l'homme et celle des animaux d'une part (Thalji et Shalaldeh, 2006) et permet une économie de la fertilisation azotée en raison de ses propriétés fixatrices d'azote atmosphérique d'autre part. Ces atouts la rendent très appréciée par les agriculteurs. La fève peut constituer une tête d'assolement très intéressante dans un programme de rotation (Halila, I995).

Du fait que $V$. faba a été cultivée depuis longtemps dans des régions agro-climatiques diverses, les variétés locales offrent de nos jours un choix d'alternatives et une grande diversité génétique. L'importance de cette richesse génétique, pour le développement de variétés améliorées est incontestable et nécessite des actions de sauvegarde en vue de diminuer les effets de l'érosion génétique. En effet, plusieurs dangers de disparition menacent ces variétés traditionnelles, à savoir la sécheresse (et/ou le changement climatique), la perte progressive du savoir traditionnel par rapport aux ressources génétiques locales, les pratiques et les utilisations traditionnelles ainsi que l'introduction des variétés étrangères (Pistrick et al., 1994; El Gazzah \& Chalabi, I995; Loumerem, I998 ; Ben Sadok, 2006).

A cet égard, des programmes de conservation des ressources génétiques de la fève ont été développés en Tunisie dans l'objectif de limiter l'impact négatif des facteurs biotiques et abiotiques sur le patrimoine génétique existant (Loumerem et al., 2004 ; Duc et al., 20I0), ainsi que pour l'obtention ultérieure d'autres variétés améliorées plus productives et mieux adaptées aux conditions pédoclimatiques des régions de culture de la fève.

L'objectif du présent travail était la caractérisation de la variabilité génétique en termes de traits morphologiques et phénologiques de I3 lignées de fève (Vicia faba L.) sélectionnées à l'Institut des Régions Arides de Médenine à partir de 42 populations locales. 
La variabilité génétique de ces lignées va être caractérisée à l'aide d'un descripteur de l'espèce Vicia faba (UPOV, 2000), ensuite évaluée et analysée par un outil statistique afin d'extraire les lignées les plus intéressantes qui serviront au programme de sélection en cours.

\section{Matériel et méthodes \\ Matériel végétal}

Les semences de fève utilisées pour effectuer ce travail de caractérisation sont descendantes de treize populations qui ont été sélectionnées parmi 42 populations locales (patrimoine phytogénétique de fèves des régions arides). Ces populations ont initialement été collectées dans les régions de Médenine, Mareth, Béni Khdache, Gabès, Tozeur, Gafsa, El Hamma, et Tataouine (Loumerem et al., 2004 ; Yahia, 2008).

\section{Conduite de l'essai}

L'essai a été conduit en 2009 sur la parcelle expérimentale de l'Institut des Régions Arides (IRA) à Mednine (Figure $\mathrm{n}^{\circ} \mathrm{I}$ ), situé dans le sud-est (latitude : $3 \mathrm{I}^{\circ}-34^{\circ}$ et longitude : $8^{\circ}-\mathrm{II}^{\circ}$ ) de la Tunisie. Le climat de cette localité est caractéristique de la zone aride, avec des températures journalières extrêmes de 6,2 à $38^{\circ} \mathrm{C}$, et des précipitations faibles allant de 50 à 150 mm de pluie par an (Le Houérou, I969; Floret \& Pontanier, I982; Le Floc'h, I986; Mzabi, I988). 

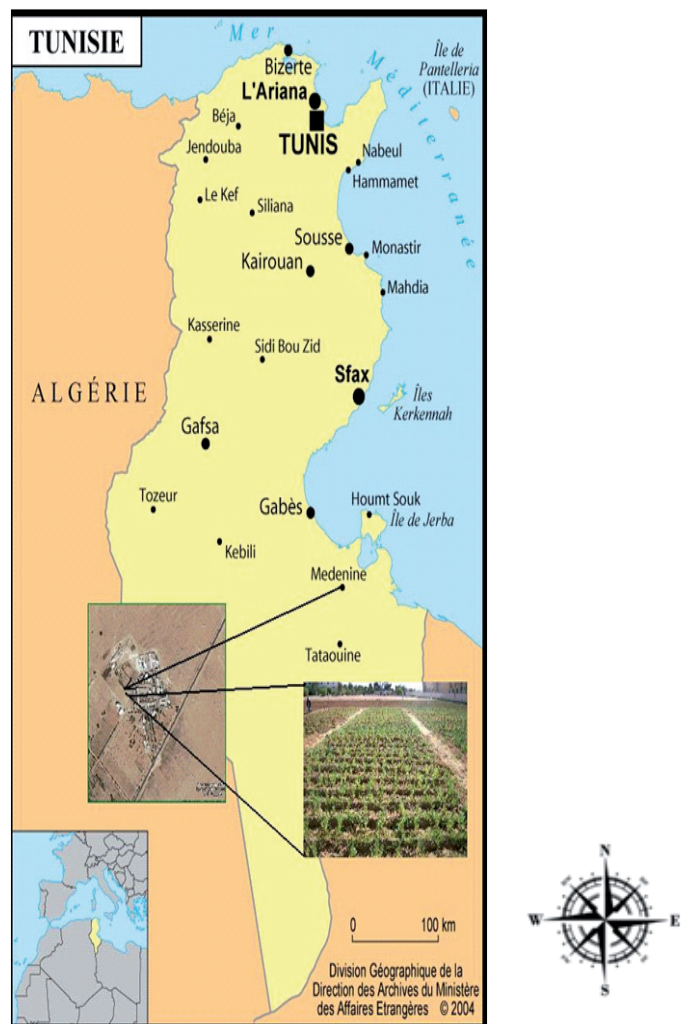

Figure 1 : La parcelle expérimentale de l'Institut des Régions Arides de Medenine.

(source : Division Géographique de la Direction des Archives du Ministère des Affaires Etrangères).

Le sol est peu profond, à texture sableuse avec une légère augmentation du taux de gypse en profondeur. Il s'agit d'un sol de type peu évolué, régosolique à faciès calcimorphe gypseux. Les teneurs en matière organique sont faibles et la perméabilité est moyenne (Floret \& Pontanier, I982 ; Escadafal, I985). Les principales caractéristiques de rétention du sol à l'IRA sont :

- teneur en eau à la saturation naturelle : $\Theta_{\mathrm{s}}=0.390 \mathrm{~cm}^{3} / \mathrm{cm}^{3}$;

- teneur en eau à la capacité au champ : $\Theta(\mathrm{pF}=2.54): \Theta_{\mathrm{cc}}=0.289 \mathrm{~cm}^{3} / \mathrm{cm}^{3}$;

- teneur en eau au point de flétrissement temporaire $\Theta(\mathrm{pF}=3.7): \Theta_{\mathrm{ft}}=0.126 \mathrm{~cm}^{3} / \mathrm{cm}^{3}$ et

- teneur en eau au point de flétrissement permanent $\Theta(\mathrm{pF}=4.2): \Theta_{\mathrm{pfp}}=0.064 \mathrm{~cm}^{3} / \mathrm{cm}^{3}$ (Yahia, 2008).

Le semis a été réalisé les 25 et 26 novembre 2009 en suivant un dispositif monofactoriel à blocs complets randomisés avec 3 répétitions. Le semis était effectué en lignes, espacées de $40 \mathrm{~cm}$ avec un inter-plant de 15 à $20 \mathrm{~cm}$ pour faciliter les travaux d'entretien et de suivi. Chaque parcelle élémentaire était constituée par deux lignes de culture. 


\section{Paramètres mesurés}

La diversité phénotypique peut être estimée à partir de données recueillies sur les individus étudiés ou sur leurs descendances (Lefort-Buson et al., I988). Elle est conditionnée par le choix des caractères. Leur nombre et leur nature posent un certain nombre de questions auxquelles seul le sélectionneur, confronté à des objectifs spécifiques peut répondre (Pernès, 1984).

La variabilité phéno-morphologique des lignées de fève a été documentée et suivie du semis jusqu'à la fin du cycle génératif (maturité des graines) par des mesures biométriques et des évaluations qualitatives sur différentes parties des plantes par lignée. Les caractères morphologiques ont été décrits en se basant sur le descripteur de l'UPOV (2000) pour l'espèce Vicia faba L. Ce descripteur comporte 34 caractères sur lesquels le travail de caractérisation peut se baser.

Dans notre cas (de figure), la caractérisation s'est basée sur 15 caractères morphologiques et 5 caractères phénologiques. Ces caractères sont de deux types, des caractères qualitatifs (Tableau I) et des caractères quantitatifs (Tableau 2). 


\begin{tabular}{|c|c|c|c|}
\hline Caractère & Code & Variabilité & Unité \\
\hline Homogénéité I & HOMI & $\begin{array}{l}\text { faible } \\
\text { moyenne } \\
\text { forte }\end{array}$ & $\begin{array}{l}3 \\
5 \\
7\end{array}$ \\
\hline Homogénéité 2 & $\mathrm{HOM}_{2}$ & & IDEM \\
\hline Intensité de couleur de la tige & ICLRTG & $\begin{array}{l}\text { faible } \\
\text { moyenne } \\
\text { forte }\end{array}$ & $\begin{array}{l}3 \\
5 \\
7\end{array}$ \\
\hline Mélanine de l'aile (fleur) & MELAIL & $\begin{array}{l}\text { absente } \\
\text { présente }\end{array}$ & $\begin{array}{l}\text { I } \\
9\end{array}$ \\
\hline Couleur de la mélanine de l'aile & CMELAIL & $\begin{array}{l}\text { jaune } \\
\text { brun } \\
\text { noir }\end{array}$ & $\begin{array}{l} \\
2 \\
3 \\
\end{array}$ \\
\hline Mélanine de l'étendard & MELETD & $\begin{array}{l}\text { absente } \\
\text { présente }\end{array}$ & $\begin{array}{l}\text { I } \\
9\end{array}$ \\
\hline Pigmentation de l'étendard & PIGETD & $\begin{array}{l}\text { absente } \\
\text { présente }\end{array}$ & $\begin{array}{l}\text { I } \\
9\end{array}$ \\
\hline $\begin{array}{l}\text { Extension de la pigmentation de } \\
\text { l'étendard }\end{array}$ & EXPIGETD & $\begin{array}{l}\text { faible } \\
\text { moyenne } \\
\text { forte }\end{array}$ & $\begin{array}{l}3 \\
5 \\
7 \\
\end{array}$ \\
\hline Port de la gousse & PRTGSS & $\begin{array}{l}\text { dressé } \\
\text { demi-dressé } \\
\text { horizontal } \\
\text { demi-retombant } \\
\text { retombant } \\
\end{array}$ & $\begin{array}{l}\text { I } \\
3 \\
5 \\
7 \\
9 \\
\end{array}$ \\
\hline Intensité de courbure de la gousse & ICRBGSS & $\begin{array}{l}\text { nulle ou très faible } \\
\text { faible } \\
\text { moyenne } \\
\text { forte }\end{array}$ & $\begin{array}{l}\text { I } \\
3 \\
5 \\
7\end{array}$ \\
\hline Intensité de couleur verte de la gousse & ICLRGSS & $\begin{array}{l}\text { faible } \\
\text { moyenne } \\
\text { forte }\end{array}$ & $\begin{array}{l}3 \\
5 \\
7\end{array}$ \\
\hline
\end{tabular}

Tableau 1 : Caractères qualitatifs étudiés sur les différentes lignées de fève.

\section{Analyses statistiques}

Les analyses statistiques ont été effectuées avec le logiciel SPSS (version I6.0). Les résultats de l'analyse de variance (ANOVA) sont considérés significatifs à un niveau $\mathrm{p}<0,5$. La comparaison des moyennes a été faite avec le test de Duncan. Le problème de cette approche est qu'elle ne permet pas de former des groupes (homogènes) de provenances 
similaires. En fait, le groupement n'était pas complet puisque le test de Duncan fournit des classements de lignées présentant des différences significatives pour un caractère donné : et là où il y a chevauchement des lignées entre plusieurs groupes ou catégories, on ne peut pas les affecter à des groupes homogènes. On doit en ce cas pousser le groupement par une analyse statistique multi-variée via la classification hiérarchique de cluster par le dressage d'un dendrogramme fournissant un groupage plus exact en tenant compte de tous les paramètres d'évaluation (Rohlf, I990).

\begin{tabular}{|l|l|l|l|}
\hline Caractère & Code & Variabilité & Unité \\
\hline Date de levée & LEVEE & I7 à 24 & nombre de jours \\
\hline $50 \%$ des plantes fleuries & FLOR50 & 59 à 8I & nombre de jours \\
\hline $50 \%$ des plantes formant des gousses & GSS50 & I07 à I22 & nombre de jours \\
\hline Hauteur de la plante & HAUTPL & I5 à 62 & cm \\
\hline Nombre des tiges/plante & NBTGE & I à 9 & nombre \\
\hline Longueur de la foliole & LNGFLL & 2 à 9 & cm \\
\hline Nombre des fleurs/racème & NBFLR & I à 9 & nombre \\
\hline Nombre des gousses/plantes & NBGSSP & I à I6 & nombre \\
\hline Nombre des graines/gousse & NBGR/G & 2 à 8 & nombre \\
\hline Poids de roo graines & PCG & I07 à 256 & g \\
\hline
\end{tabular}

Tableau 2: Caractères quantitatifs étudiés sur les différentes lignées de fève.

\section{Résultats et discussion \\ Analyse des caractères qualitatifs}

Homogénéité du développement

La caractérisation de l'homogénéité de développement des individus au sein d'une même lignée a été effectué en 2 phases. La première phase se situait à 84 jours après semis (stade floraison, figure 2), et la deuxième phase à I40 jours après semis (fructification). Au moment de la première évaluation (HOMI), seulement 45,4 \% des lignées ont été qualifiées homogènes. La classification des lignées selon ce critère d'homogénéité les regroupe en 4 classes :

- la première classe est constituée par les lignées I, 5, 6, et io qui représentent 29,9\% du total des individus dont $63,6 \%$ sont homogènes et $36,4 \%$ très homogènes ;

- les lignées 2, 3, 4, 7, I2 et I 3 forment la deuxième classe et représentent $46,7 \%$ du total avec $5 \mathrm{I} \%$ des individus très homogènes ;

- la lignée 8 constitue à elle seule une classe, la troisième avec un taux d'homogénéité de $66,7 \%$; et

- enfin, la quatrième classe représentée par les lignées 9 et II qui sont homogènes à $36,1 \%$ seulement. 


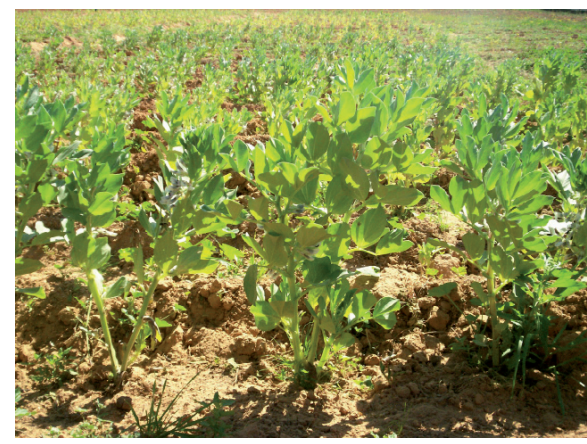

Figure 2 : Homogénéité de plantes d’une lignée au stade floraison.

Lors de la seconde évaluation de l'homogénéité ( $\left.\mathrm{HOM}_{2}\right)$, on a répertorié plus d'individus homogènes par rapport au premier passage. En effet, le pourcentage de lignées homogènes a augmenté à 56,7\%. La lignée 13 qui comprend le plus (72,9\%) d'individus homogènes ; elle est suivie par les lignées $\mathrm{I}, 3,6$, et Io avec $68,5 \%$ d'individus homogènes, ensuite 7, 9 et I 2 avec 6I,I \% d'homogénéité. Les lignées les moins homogènes sont 2, 4, 5, 8 et II avec seulement 4I,9 \% d'individus homogènes.

L'intensité de la couleur verte de la tige (ICLRTGE)

Pour le caractère (ICLRTGE), 25,7\% des individus ont une couleur vert-claire à faible intensité et 74,3\% sont à tiges vertes d'intensité moyenne. Trois groupes se distinguent:

- le premier groupe est constitué par les lignées I, 2, 4, 6, 9, II, I2 et I3 et présente $60,7 \%$ du total des individus. Dans ce groupe, 20,8\% des individus présentent une couleur verte de faible intensité au niveau de leur tige et 79,2 \% possèdent des tiges à intensité de couleur moyenne;

- les lignées 3 et ro forment le deuxième groupe et représentent $15,5 \%$ du total des individus. Dans ce groupe, $37,8 \%$ des individus ont des tiges à faible intensité de couleur et $62,2 \%$ des tiges vertes d'intensité moyenne ; or que

- le troisième groupe est composé des lignées 5, 7 et 8 avec 30,I \% des individus à tiges vertes de faible intensité et $69,9 \%$ à tiges vertes d'intensité moyenne.

Longueur de la fleur (LNGFLR)

Sur 1576 plantes de fève, I2,I \% ont de petites fleurs, 87,5\% ont des fleurs à longueur moyenne or qu'il n'y en a que 0,4 \% qui ont de longues fleurs (Figure 3). Selon ce critère (LNGFLR), les lignées étudiées se classent comme suit :

- les lignées I et 5 avec $17,4 \%$ des individus à petites fleurs, 80,5\% à fleurs moyennes et 2,I \% à fleurs longues ;

- les lignées 2, 8 et I 3 qui représentent $23,4 \%$ du total, ont I2,4 \% de leurs individus à petites fleurs et $87,6 \%$ à fleurs moyennes ;

- les lignées 3, 6, 9 et II avec 5,2\% des individus à petites fleurs et 94,8\% à fleurs moyennes ; 
- la lignée 4 qui représente 8,I \% du total des individus avec I0,9\% des individus à petites fleurs, $88,3 \%$ à fleurs moyennes et o, $8 \%$ à fleurs longues; et finalement

- les lignées 7 , I0, et $\mathrm{I} 2$ qui regroupent $\mathrm{I} 7,7 \%$ de plantes à petites fleurs, et $82,3 \%$ à fleurs moyennes.

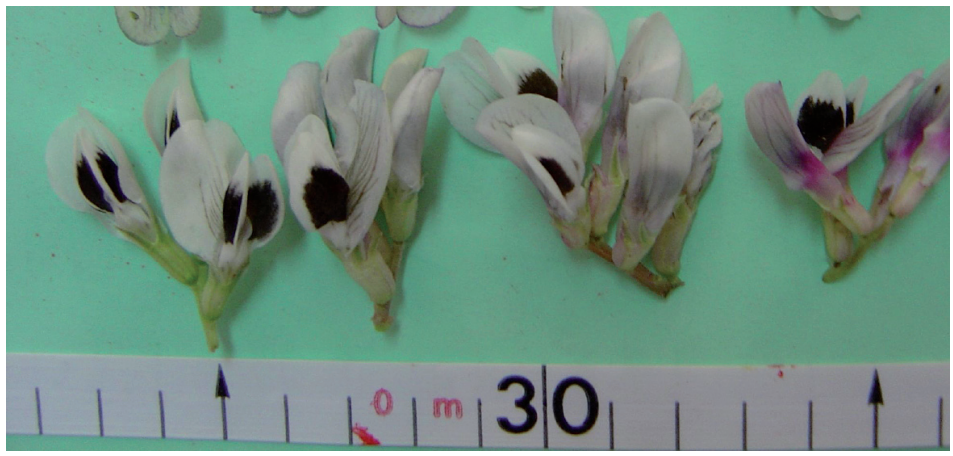

Figure 3 : La variabilité de la longueur des fleurs.

La tache de mélanine de l'aile

Presque 99,6 \% des plantes de fève suivies présentent des taches de mélanine sur l'aile de leur fleur (MELAIL).

La couleur de la tache de mélanine de l'aile

Concernant ce caractère (CMELAIL), deux couleurs ont été observés, qui sont le noir et le brun : 92,8\% des plantes des lignées étudiées ont une tache de mélanine de l'aile noire et uniquement $7,2 \%$ ont une tache de mélanine de l'aile brune.

La tache de mélanine de l'étendard

Soixante neuf pour cent des individus de fèves suivis présentent des taches mélaniques sur l'étendard de leurs fleurs (MELETD).

\section{Pigmentation de l'étendard}

Environ $52 \%$ des lignées étudiées ont des fleurs à étendards pigmentés (PIGETD), le reste est dépourvu de pigmentation. Selon ce critère de classification, on regroupe les lignées suivies en quatre groupes :

- les lignées I et 6 , qui constituent 7I,2 \% des individus, présentent des fleurs à étendard pigmenté;

- les lignées 2, 3, 9 et Io dont la présence de la pigmentation de l'étendard chez les individus est de $58,5 \%$;

- les lignées 4, 5, 7, II et I 3 dont 53,8\% des individus sont dépourvus de pigmentation de l'étendard; et

- les lignées 8 et $\mathrm{I} 2$ ont $67, \mathrm{I} \%$ de leurs individus à étendard non pigmenté. 
Extension de la pigmentation de l'étendard (EXPIGETD)

Ce caractère (EXPIGETD) concerne l'extension de la pigmentation de l'étendard des fleurs ; 48,4 \% des individus sont sans pigmentation. Le reste des individus est à 4I, 3 \% à pigmentation d'étendard à faible extension et à Io, $3 \%$ d'étendards à pigmentation moyennement exténue. Suivant ce critère de classification, les lignées étudiées forment deux grands groupes ( $48,4 \%$ sans pigmentation et 5I, $6 \%$ avec pigmentation).

Port de la gousse (PRTGSS)

Pour ce caractère (PRTGSS), 20,6\% des individus présentent des gousses dressées, $34,2 \%$ des gousses demi-dressées, I4, $6 \%$ des gousses horizontales, $25, \mathrm{I} \%$ des gousses demi-retombants et $5,5 \%$ des gousses retombantes. Les groupes obtenus selon ce caractère sont au nombre de 8 :

- les lignées I, 2, et 6 forment un groupe dont 50,3\% des individus ont des gousses dressées, 40,7\% des gousses semi-dressées, 6,2 \% des gousses horizontales, 2,2 $\%$ des gousses demi retombantes et $0,6 \%$ des gousses retombantes;

- les lignées 4, 7, et 8 constituent un groupe d'individus dont les ports des gousses sont à I $6,4 \%$ dressés, 50,4\% semi-dressés, 20,1\% horizontaux, II,7\% semi-retombants et $\mathrm{I}, 3$ \% retombants;

- les lignées Io et II se réunissent en groupe dont les plantes ont des gousses à 57,6\% semi-retombants ; et

- les autres lignées forment, chacune en elle seule, un groupe à part; la lignée 3 présente $32,3 \%$ d'individus à gousses dressées et 59,3\% d'individus à gousses semidressées; la lignée 5 est à 36,4\% d'individus à gousses semi-dressées; les lignées 9 , I2 et I 3 englobent, chacune dans son propre groupe, respectivement 54,I \%, 34,2 \% et $6 \mathrm{I}, 9 \%$ de plantes à gousses semi-retombantes.

Intensité de courbure des gousses (ICRBGSS)

Les gousses de fève observées présentent une large variabilité en termes de courbure (ICRBGSS). Cette courbure est nulle ou très faible chez 20,4\% des individus; faible chez 46,6 \%; moyenne chez 21,3\% et forte chez 3,7\% des individus (Figure 4 ).

La classification des lignées selon ce critère permet de dégager 9 groupes à savoir:

- les lignées I et 2 présentées par des plantes qui développent des gousses dont 43,2 $\%$ sont à courbure nulle ou très faible, $40 \%$ à courbure faible, $7,4 \%$ à courbure moyenne et $0,4 \%$ à forte courbure;

- les lignées 3 et 6 englobent des individus dont les gousses sont de 40,4\% à courbure nulle ou très faible, $66,4 \%$ à courbure faible et $4,2 \%$ à courbure moyenne;

- les lignées 4,5 , et 7 comportant des individus aux gousses de courbures très faibles pour $33,6 \%$ des plantes, faibles pour $53,6 \%$, moyennes pour II, $7 \%$ et forte pour I,I \% des individus;

- les lignées restantes, forment chacune un groupe à part, avec la lignée 8 avec $44,2 \%$ d'individus à gousse de courbure nulle, et 4I,I \% à courbure faible; la lignée 9 avec 43,4\% d'individus dont les gousses sont à courbure moyenne; 
la lignée io avec 47,I \% des plantes aux gousses faiblement courbées et 30,6\% moyennement courbées; la lignée II à 5I,3\% des plantes ayant des gousses à courbure moyenne et 3I,9 \% à courbure faible; la lignée $\mathrm{I} 2$ avec 47,2 \% des individus aux gousses à faible courbure et enfin la lignée $\mathrm{I} 3$ avec $48,3 \%$ des plantes ayant des gousses à courbure moyenne.

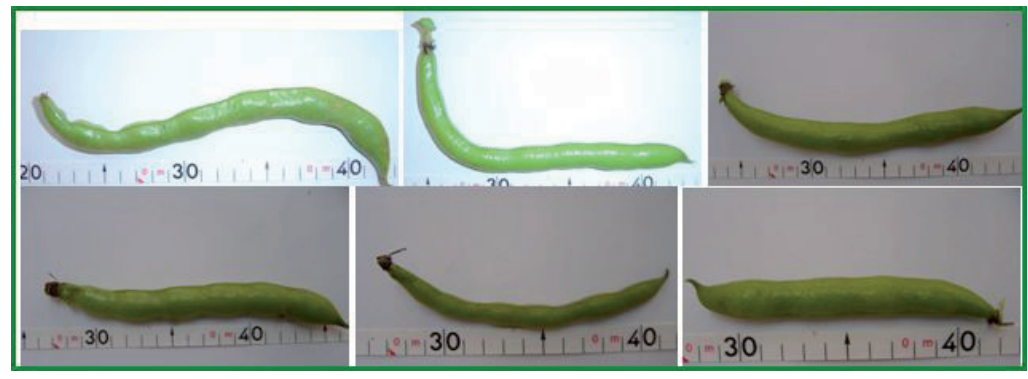

Figure 4 : Intensité de courbure des gousses des lignées étudiées.

Intensité de la couleur verte des gousses (ICLRGSS)

La couleur verte de la gousse peut être claire, moyenne ou bien foncée. On a remarqué que $18, \mathrm{I} \%$ des individus ont des gousses de couleur vert-claire, 74,9 \% ont des gousses vertes et $6,8 \%$ des gousses vert-foncées.

L'analyse de tous ces paramètres qualitatifs montre que les caractéristiques des lignées de fêve locales étudiées répondent en grande partie à celles de la plupart des variétés représentées par le germplasme de cette espèce disponible à l'ICARDA (Perrino et al., I99I).

\section{L'analyse des caractères quantitatifs}

Dix caractères quantitatifs ont été analysés chez les lignées de fève (tableau 2).

Nous avons adopté, lors de notre étude, le modèle d'analyse de variance à un seul facteur de classification (effet lignée), pour essayer de séparer dans la variabilité totale observée celle due aux facteurs inter-lignées de celle due aux facteurs environnementaux et génétiques intra-lignée (variation résiduelle). Le programme utilisé pour cette analyse est le programme SPSS version $\mathrm{I} 6.00$.

Les résultats de l'analyse de variance à un seul facteur de classification sont présentés dans le tableau 4. Ce tableau résume l'ensemble des résultats obtenus pour l'ensemble des lignées sélectionnées à l'Institut des Régions Arides pour la création de nouvelles variétés plus productives et mieux adaptées à ces régions arides. 


\begin{tabular}{|c|c|c|c|c|c|}
\hline Variable & ddl & SCE & CM & Fcal & Sig. \\
\hline LEVEE & $\mathrm{I} 2$ & 966,003 & 80,500 & 39,167 & HS \\
\hline FLOR50 & $\mathrm{I} 2$ & 10263,156 & 855,263 & 27,660 & HS \\
\hline GSS & $\mathrm{I} 2$ & 23382,920 & I948,577 & 85,172 & $\mathrm{HS}$ \\
\hline HAUTPL & $\mathrm{I} 2$ & 7178,255 & 598,188 & $\mathrm{II}, \mathrm{I} 82$ & HS \\
\hline NBTGE & $\mathrm{I} 2$ & 200,710 & I6,726 & I0,793 & HS \\
\hline LNGFLL & $\mathrm{I} 2$ & I88,940 & 15,745 & 16,563 & HS \\
\hline NBFLRR & $\mathrm{I} 2$ & 176,796 & $\mathrm{I} 4,733$ & $\mathrm{I} 4,057$ & HS \\
\hline NBGSSP & $\mathrm{I} 2$ & 542,760 & 45,230 & 13,389 & HS \\
\hline NBGRPGSS & $\mathrm{I} 2$ & I74I, 608 & $\mathrm{I} 45, \mathrm{I} 34$ & 287,562 & HS \\
\hline PDSCGR & $\mathrm{I} 2$ & 414639,096 & 34553,258 & 56,736 & HS \\
\hline
\end{tabular}

Tableau 3: Analyse de la variance à un critère de classification pour les caractères quantitatifs étudiés.

HS : hautement significatives

Fcal : la valeur de $\mathrm{F}$ calculée

$\mathrm{ddl}$ : nombre de degrés de liberté

$\mathrm{CM}$ : carrés moyens

SCE : somme des carrés des écarts

Les valeurs de F calculées (Fcal) démontrent que pour tous les caractères étudiés, les différences inter-lignées sont hautement significatives (HS). Les lignées sont donc statistiquement différentes. Ceci n'est pas étonnant, étant donné que d'une part, les lignées ont été choisies à partir de différentes populations, et que d'autre part, on a cherché à garder la plus grande diversité.

Nous remarquons que pour certains caractères, Fcal présente des valeurs élevées, par exemple: le nombre de graines par gousse $(\mathrm{F}=287,562)$, la date de formation des gousses chez $50 \%$ des individus $(85,172)$ et le poids de cent graines $(56,736)$. Il s'agit alors de caractères qui différencient le plus les lignées.

Pour estimer la ressemblance entre les lignées, nous avons adopté la méthode de Duncan qui permet de chercher des groupes de lignées homogènes, en considérant les caractères pour lesquels les valeurs de F se sont avérées significatives.

Le test Duncan, procédure de comparaison multiple, permet de comparer toutes les paires de moyennes, en contrôlant le risque alpha $(\alpha)$ général à un niveau défini (Dagnelie, I965).

Pour un caractère donné, les moyennes des lignées sont comparées par le test Duncan à $5 \%$. Les résultats de ces tests ont été rassemblés dans le tableau 5 . 
Caractérisation phéno-morphologique de quelques lignées de fève (Vicia faba L.)

La date de levée (LEVEE)

Pour les durées de levée, elles sont comprises entre 17 et 24 jours à partir de la date de semis. Le test de DUNCAN (tableau 5) a permis de distinguer 6 groupes :

- un premier groupe constitué par les lignées II, 8, 3, 4, et 5 et présentant une moyenne de 17,33 jours ;

- un deuxième groupe : formé par les lignées 3, 4, 5 et 7 ;

- un troisième groupe : comporte les lignées 7 , I et I2 ;

- un quatrième groupe : les lignées 6 et 9 ;

- un cinquième groupe : les lignées 2 et I3 ; et

- un dernier groupe est formé par la lignée Io avec une moyenne de I9,87 

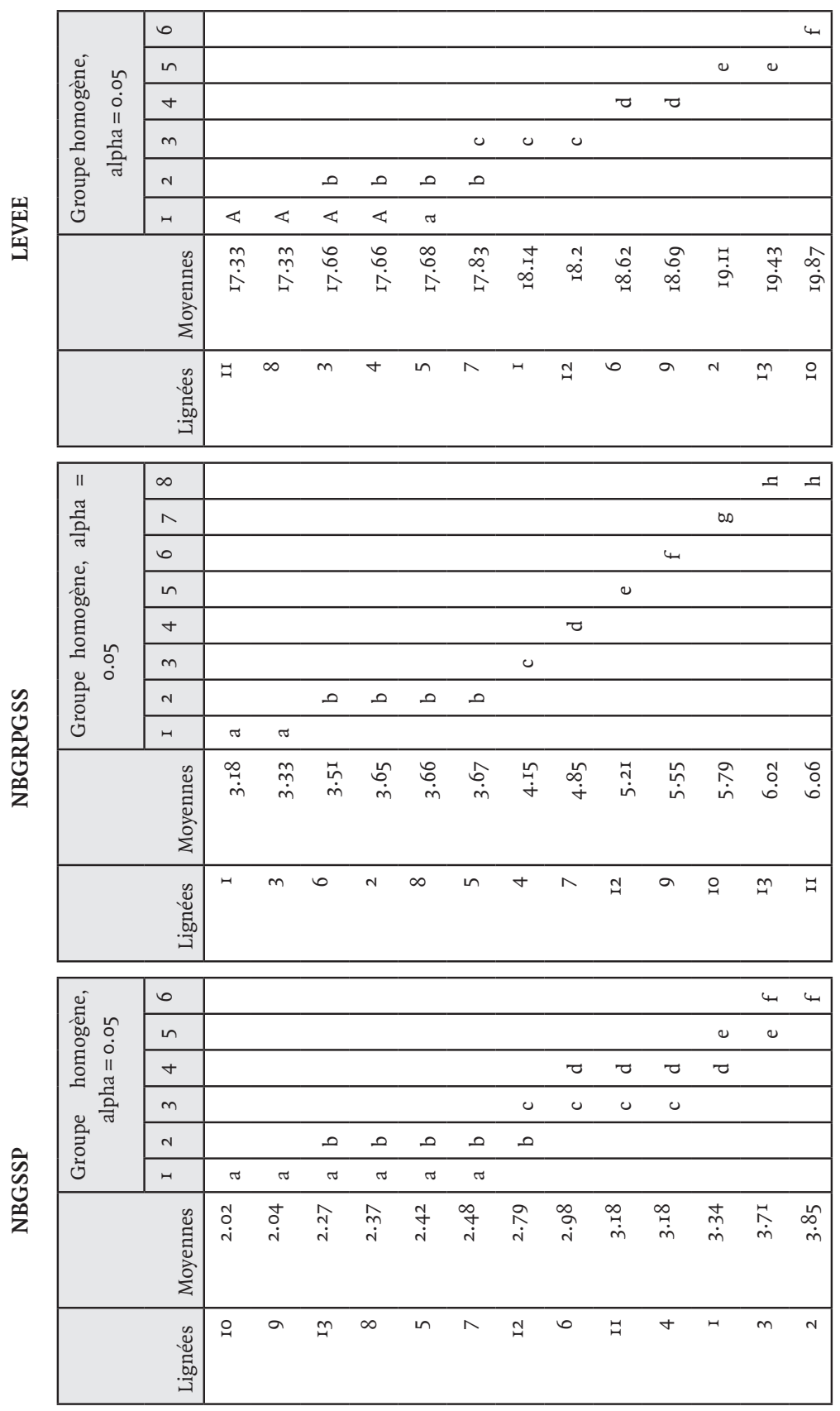
Caractérisation phéno-morphologique de quelques lignées de fève (Vicia faba L.)

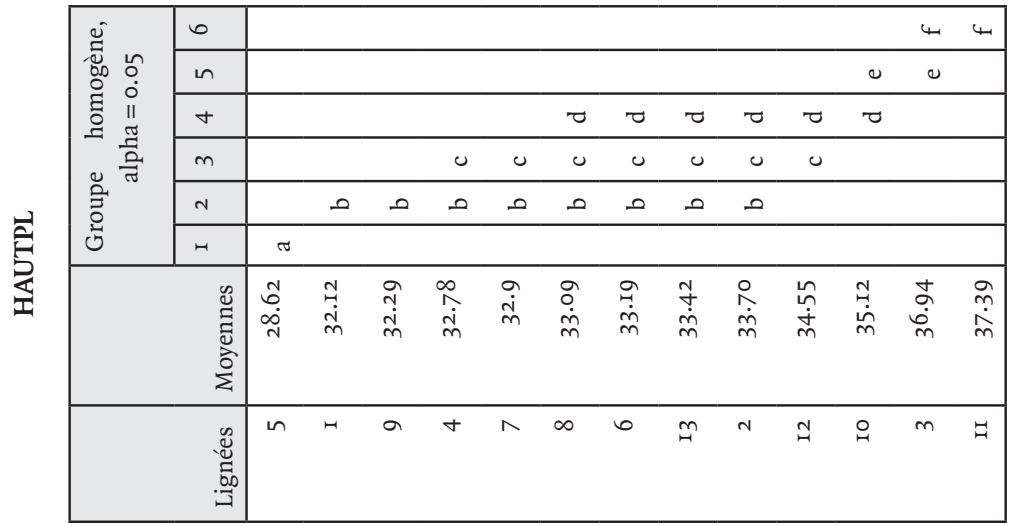

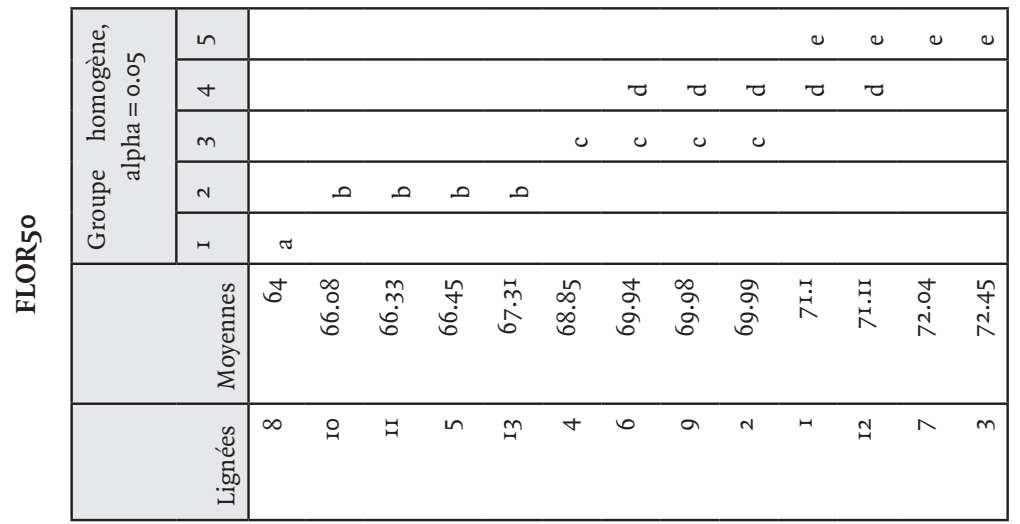

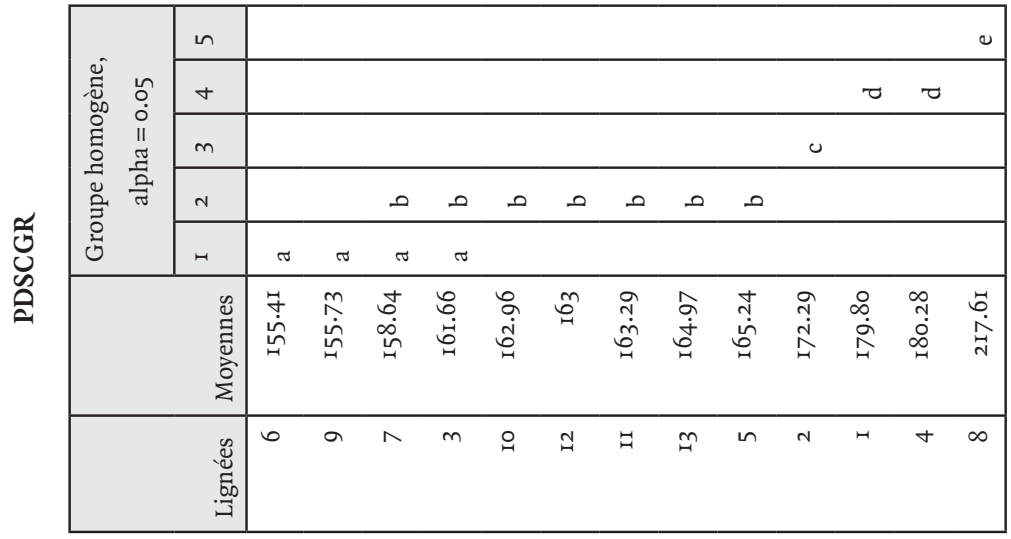



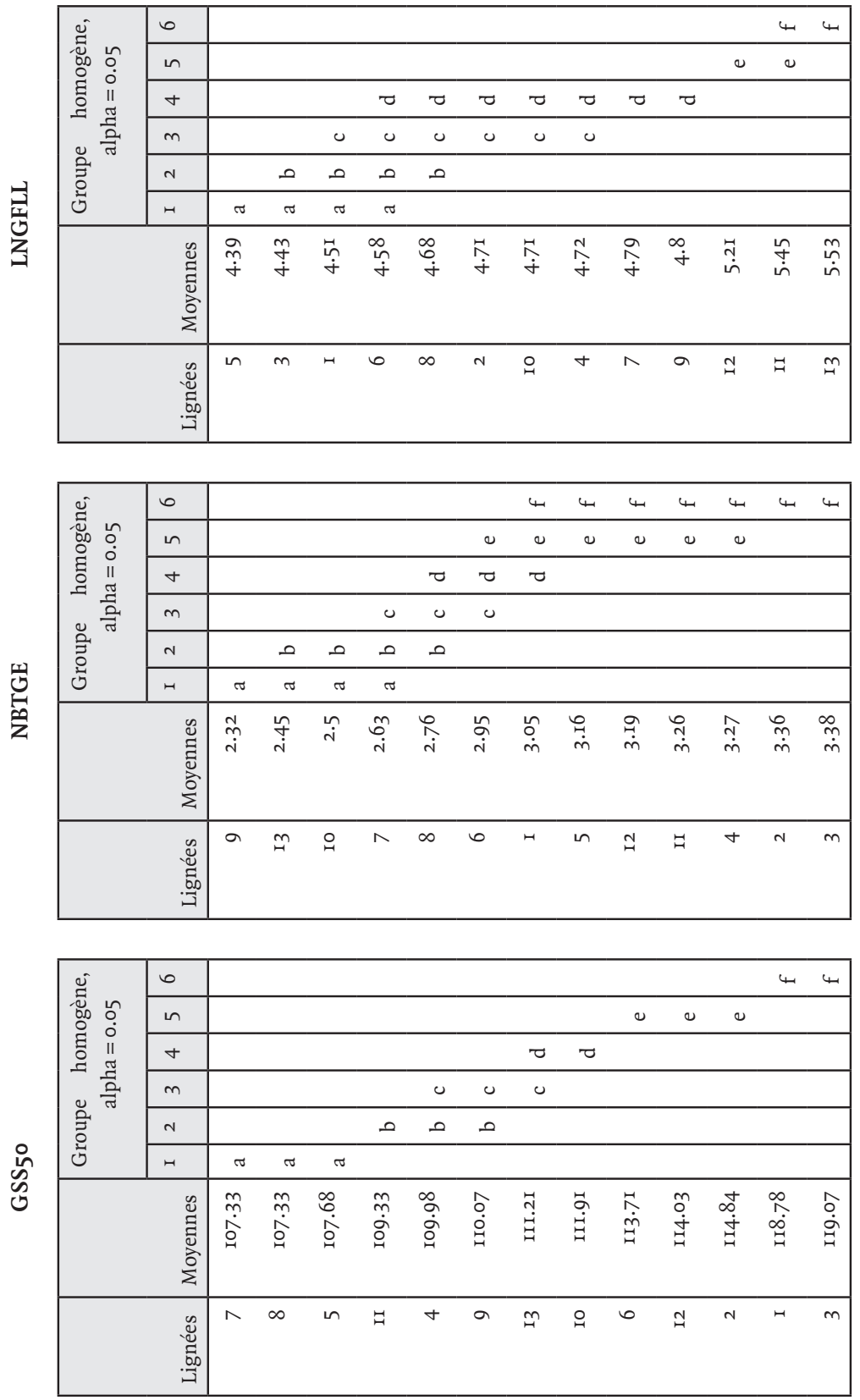

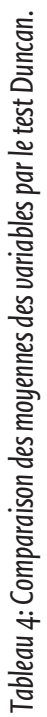


La date à $50 \%$ de floraison (FLOR50)

La floraison de 50 \% des individus d'une même lignée, nous a permis de regrouper les lignées testées en 5 groupes :

- un premier groupe représenté par la lignée 8 avec une date de floraison moyenne de 64 jours ;

- un deuxième groupe : qui comporte les lignées Io, II, 5 et I3 avec une valeur FLOR 50 moyenne de 66 à 67 jours ;

- un troisième groupe : les lignées 4, 6, 9 et 2 ( 67 à 70 jours);

- un quatrième groupe : les lignées 6, 9, 2, I et I2 (70 à 72 jours); et

- un dernier groupe composé des lignées I, I2, 7 et 3 (7I à 73 jours).

La date de formation des gousses chez $50 \%$ des individus (GSS)

Les dates de formation des gousses varient selon les lignées de I07 à I22 jours à partir de la date de semis :

- le premier groupe est formé par les lignées 7,8 et 5 avec des moyennes respectives de 107,33 jours pour les deux premières et 107,68 jours pour la lignée 5 ;

- le deuxième groupe : les lignées II, 9 et 4 ;

- le troisième groupe : les lignées 9, 4 et 13 ;

- un quatrième groupe : les lignées 13 et Io ;

- le cinquième groupe : les lignées 6, I2 et 2 ; avec des moyennes respectives de II 3,7 I jours, II 4,03 et II 4,84 jours ; et

- le dernier groupe : les lignées I et 3 avec des moyennes respectives II 8,78 jours et II 9,07 jours.

La hauteur de la plante (HAUTPL)

Les groupes ressortis du test de Duncan sont les suivants :

- un premier groupe représenté par la lignée 5 constituée par les individus les plus petits avec une taille moyenne de $28,62 \mathrm{~cm}$;

- un deuxième groupe constitué par les lignées I et 9, 4, 7, 8, 6, I3 et 2 ;

- un troisième groupe constitué par les lignées 4, 7, 8, 6, I3, 2 et I2;

- un quatrième groupe constitué par les lignées 8,6 , I3, 2, I2 et Io;

- un cinquième groupe formé par les lignées Io et 3 ; et

- un dernier groupe formé par les lignées 3 et II qui englobent les individus de plus grande taille $(36,94$ et $37,39 \mathrm{~cm}$ respectivement).

Nombre de tiges par plante (NBTGE)

Les lignées étudiées renferment des plantes dont le nombre de tiges varie entre 2 et 9. Six groupes sont distingués :

- les lignées 9, I3, Io et 7 constituent un premier groupe avec le nombre moyen de tiges par plante la plus faible ;

- le deuxième groupe est constitué par les lignées I3, Io, 7 et 8 avec le nombre moyen de tiges par plante allant de 2,45 à 2,67; 
- les lignées 7, 8 et 6 forment le troisième groupe ;

- les lignées 8,6 , et I forment le quatrième groupe ;

- le cinquième groupe comporte les lignées 6, I, 5 , I2, II et 4 ; et

- les lignées 2 et 3 forment le dernier groupe avec les moyennes les plus élevées ( 3,38 tiges/plante).

Il est noté que la plupart de ces lignées ont un faible nombre de tiges par plante. Ceci est probablement dû à leur origine géographique. En effet, en étudiant les collections de fève, formant le germplasme disponible à l'ICARDA, Perrino et al. (I99I) ont constaté que la plupart des accessions ayant moins de 5 tiges/plante sont originaires de l'Egypte alors que la plupart de celles ayant plus de 15 tiges/plante proviennent de la Grèce.

Longueur de la foliole (LNGFLL)

La longueur des folioles des fèves peut varier de 2 à $9 \mathrm{~cm}$ (figure 5). En moyenne, les plantes ayant les folioles les moins longues appartiennent à la lignée 5 avec une moyenne de 4,39 cm alors que la moyenne la plus importante était observée chez la lignée I 3 $(5,53 \mathrm{~cm})$.

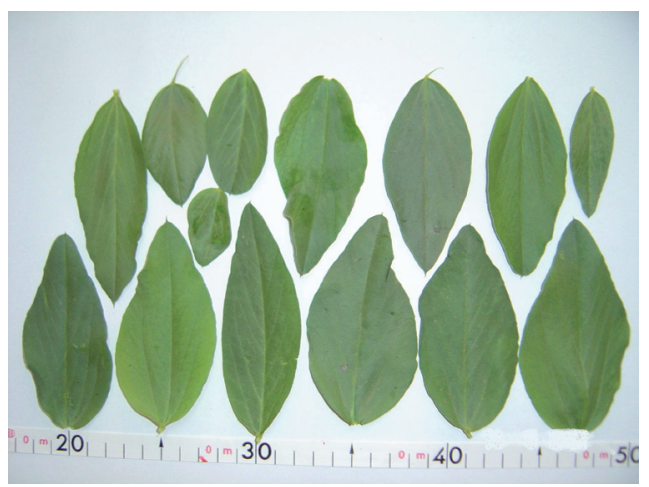

Figure 5 : La variabilité de la longueur des folioles selon les lignées.

Nombre de fleurs par racème (NBFLRR)

Le nombre de fleurs par racème varie de I à 9. La lignée I2 est caractérisée par des individus dont le nombre de fleurs par racème est le moins élevé avec une moyenne de 2,7I alors que la lignée 2 renferme les individus dont le nombre de fleurs par racème est le plus important avec une moyenne de 3,74 fleurs.

Nombre de gousses par plante (NBGSSP)

La variabilité en termes de NBGSSP prend une valeur minimale de zéro et une valeur maximale de 16 gousses par plante. On distingue les groupes suivants :

- un groupe constitué par les lignées Io, 9, I3, 8, 5 et 7 avec des moyennes variant entre 2,02 et 2,48 ;

- un groupe constitué par les lignées $\mathrm{I}_{3}, 8,5,7$, et I2 ; 
- un groupe constitué par les lignées I2,6, II, et 4 ;

- un groupe formé des lignées 6, II, 4, et I ;

- un groupe qui englobe les lignées I et 3 ;

- un groupe constitué par les lignées ayant les moyennes les plus grandes et qui sont la lignée 3 avec une moyenne de 3,7I et la lignée 2 avec 3,85 de moyenne.

Nombre de graines par gousse (NBGRPGSS)

Le nombre de graines par gousse varie chez les lignées étudiées entre 2 et 9 graines. On distingue 8 groupes :

- les lignées I et 3 avec les moyennes les plus petites, respectivement de 3,I8 et 3,33 graines ;

- les lignées 6, 8, 2 et 5 avec des moyennes respectives de 3,5I ; 3,65; 3,66 et 3,67 graines;

- les lignées I 3 et II avec des moyennes respectives de 6,02 et 6,06 et présentant les moyennes maximales; or que

- les autres lignées constituent chacune un groupe à part.

Les lignées étudiées présentent un nombre de graines par gousse comparable à la plupart des variétés de fêve cultivées en Tunisie et dans certains autres pays. En effet, l'étude de ce paramètre chez 753 accessions de fève obtenues à partir de 15 pays européens, méditerranéens et asiatiques, a montré qu'elles ont toutes un nombre de graines par gousse variant de i à 6 (Perrino et al., I99I).

Poids de cent graines (PDSCGR)

Pour ce caractère, les lignées étudiées peuvent avoir des poids de cent graines allant de 107 à $256 \mathrm{~g}$. Les groupes différenciés par le test de Duncan sont :

- les lignées 6, 9, 7 et 3 qui présentent les moyennes les plus petites qui varient entre $\mathrm{I} 55,4 \mathrm{Ig}$ et $\mathrm{I} 6 \mathrm{I}, 66 \mathrm{~g}$;

- les lignées 7,3, I0, I2, II, I3 et 5 sont réunies avec des moyennes qui ne représentent pas une différence significative, et varient entre 158,64 et $165,24 \mathrm{~g}$;

- la lignée 2 avec une moyenne de 172,29 g ;

- les lignées I et 4 ont des moyennes respectives de I79,80 et I80, $28 \mathrm{~g}$; or que

- la lignée 8 a la moyenne la plus grande de 2I7,6I; c'est au sein de cette lignée que le poids maximum est de $256 \mathrm{~g}$.

Ceci est conforme avec ce qui a été noté chez la plupart des accessions de fève mentionnées plus haut où pour le paramètre "poids de cent graines ", la valeur a varié de IzO à 2039 (Perrino et al., I99I).

La comparaison des moyennes par le test Duncan nous a permis d'effectuer un groupement entre les lignées selon les caractères étudiées. (tableau 5). 


\begin{tabular}{|c|c|c|}
\hline Lignées groupées & Caractère de classification & Description \\
\hline \multirow[t]{3}{*}{ Io, II et I3 } & Date $50 \%$ floraison & Floraison précoce \\
\hline & Poids de cent graines & PDSCGR moyen \\
\hline & Nombre de graines par gousse (II et I3) & NBGRPGSS les plus importants \\
\hline \multirow[t]{2}{*}{8 et 5} & Date $50 \%$ gousses & Fructification précoce \\
\hline & Nombre de graines par gousse & NBGSSP moyen \\
\hline \multirow[t]{2}{*}{2 et 3} & Nombre de tiges par plante & NBTGE les plus importants \\
\hline & Nombre de gousses par plante & NBGSSP les plus élevés \\
\hline \multirow[t]{2}{*}{6 et 9} & Date de levée & Levée moyenne \\
\hline & Poids de cent graines & PDSCGR faible \\
\hline
\end{tabular}

Tableau 5 : Groupes obtenus suite au test de Duncan.

Nous remarquons plusieurs types de chevauchements dans le regroupement des lignées, en particulier pour le nombre de graines par gousse, la longueur de la foliole, le nombre de tiges par plante et la hauteur de la plante.

D'une façon générale, l'évaluation de la diversité morphologique varie selon les caractères considérés. Il est donc impératif d'étudier la structure de cette diversité en analysant conjointement l'ensemble des caractères.

C'est ce que l'on a fait au travers d'une classification hiérarchique qui réunit les lignées les plus semblables. Elle utilise les dissemblances ou distances entre objets lors de la formation des classes (Pernès, I984; Dagnelie, I986), (figure 6). 
Caractérisation phéno-morphologique de quelques lignées de fève (Vicia faba L.)

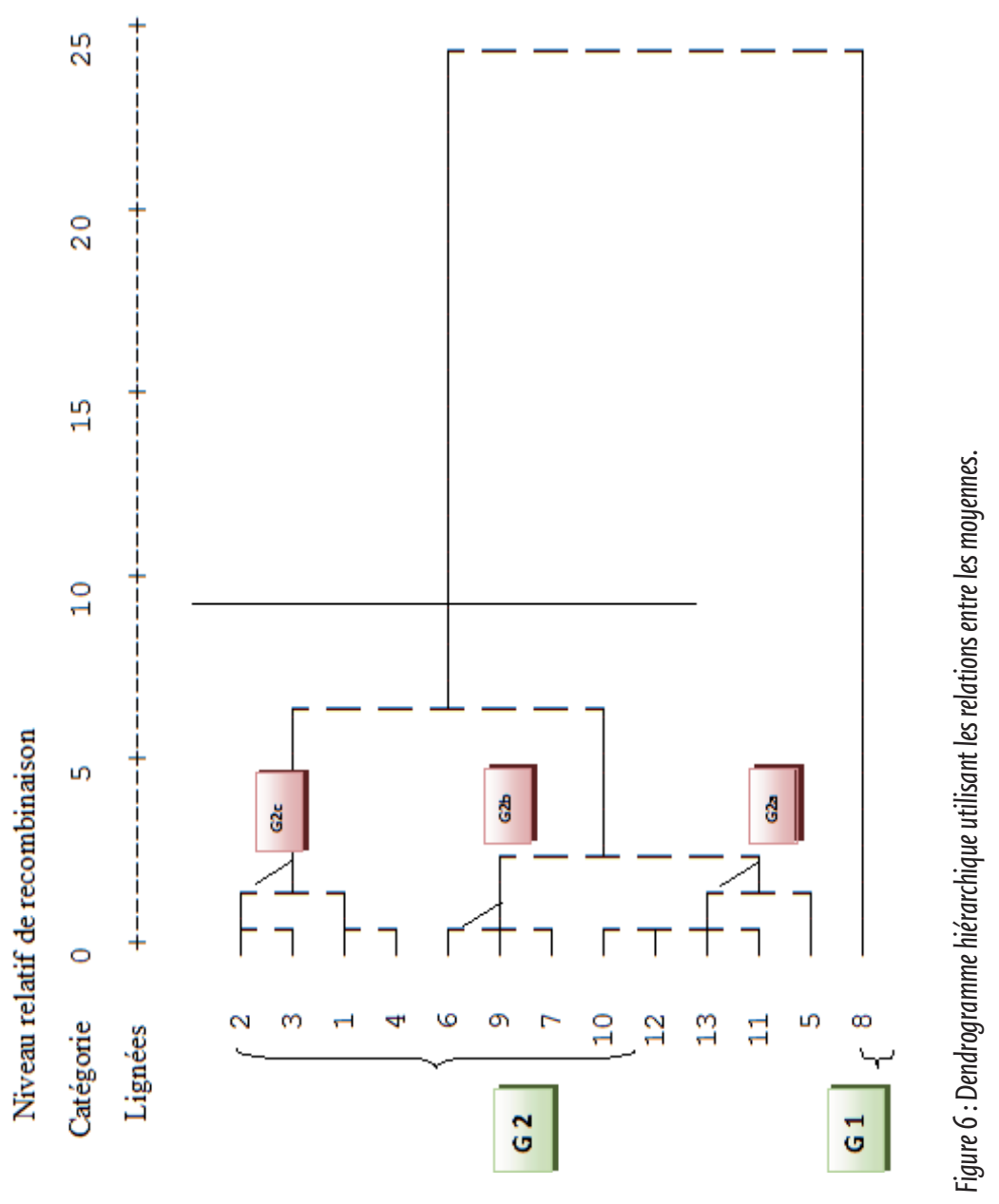




\section{L'analyse statistique multi-variée de la diversité des lignées de fève étudiées}

Le dendrogramme résultant de la classification hiérarchique des lignées (distances euclidiennes sur les moyennes des caractères étudiés) montre deux groupes de lignées qui s'individualisent avec notamment un groupe constitué par la lignée 8 uniquement :

- la lignée 8 caractérisée par une précocité importante et une valeur du poids de cent graines la plus élevée ;

- le reste des lignées qui forment le deuxième groupe sont plus ou moins tardives.

Ce deuxième groupe se subdivise en trois sous-groupes :

- un premier sous-groupe : constitué par les lignées 5, II, I3, I2 et io, qui sont similaires du point de vue " nombre de fleurs par racème " et " poids de cent graines " et caractérisé par :

- le nombre de fleurs par racème le plus faible 2,7I à 2,98, et

- un poids de cent grains moyen de I62,96 à I65,24 g.

Puis un deuxième sous-groupe formé par les lignées 7,9 et 6 qui se distinguent principalement par :

- la date 50\% floraison variant de 70 à $7 \mathrm{I}$ jours après le semis en moyenne ;

- la hauteur de plante dont la moyenne varie de 32 à $33 \mathrm{~cm}$; et

- la longueur de la foliole dont la moyenne varie de 4,5 à 4,8 cm.

Finalement un troisième sous-groupe constitué des lignées 4, I, 3 et 2; ces lignées présentent des similitudes au niveau du :

- le nombre de gousses par plantes le plus élevé de 3,18 et 3,85 gousses ;

- le nombre de tiges par plante le plus élevé de l'ordre de 3,3 tiges par plante en moyenne; et

- le nombre de fleurs par racème le plus élevé avec une valeur de 3,43 à 3,74 en moyenne. La comparaison des moyennes et la classification hiérarchique ont permis de classer les lignées entre eux et de construire un dendrogramme donnant les distances euclidiennes des moyennes des caractères étudiés. Les résultats obtenus montrent que chaque groupe a réuni les lignées les plus semblables. Ceci permet de penser que les lignées ainsi groupées ont vraisemblablement des génotypes voisins, au moins pour les caractéristiques étudiées.

\section{Conclusion}

La caractérisation phéno-morphologique de I3 lignées de fève sélectionnées à l'Institut des Régions Arides de Médenine, d'une part montre une variabilité phénotypique importante pour la majorité des caractères étudiés. D'autre part, les lignées se regroupent très peu pour certains caractères tels que le nombre de graines par gousse, le nombre de gousses par plante et le poids de cent graines. Ces lignées avaient toutes obtenu un score élevé aux tests d'évaluation morphologique basée sur des observations sur terrain. Elles représentent en plus la diversité génétique des populations de fève locale. 
La synthèse de l'ensemble des analyses effectuées nous a permis d'effectuer la classification suivante :

- la lignée 8 est la plus homogène au stade floraison avec un taux d'homogénéité de $66,7 \%$, elle se caractérise par une levée et une floraison plus précoces. Cette lignée présente aussi le poids de cent graines le plus élevé or que le nombre de gousses par plante reste peu important.

- le reste des lignées qui sont plus ou moins tardives est réparti en trois sousgroupes :

- un premier sous-groupe constitué par les lignées 5, II, I3, I2 et ro qui sont similaires du point de vue « nombre de fleurs par racème » et « poids de cent graines" ;

- un deuxième sous-groupe formé par les lignées 7, 9 et 6 est le plus homogène du point de vue " date 50\% floraison ", " hauteur de plante " et « longueur de la foliole".

- un troisième sous-groupe constitué des lignées 4, I, 3 et 2 présente le nombre le plus élevé de gousses par plante, le nombre de tiges par plante le plus élevé et le nombre de fleurs par racème le plus élevé.

Ce dernier sous-groupe peut servir comme un matériel génétique de base pour la recombinaison génétique, la production de nouveaux géniteurs et pour une sélection de nouvelles variétés.

Ces lignées homogènes devraient être conservées et valorisées pour les programmes d'amélioration variétale de l'espèce Vicia faba par des sélections successives jusqu'à l'obtention d'une variété synthétique plus productive et mieux adaptée aux conditions arides du sud tunisien.

\section{Références}

Ben Sadok, I. (2006). Etude de la variabilité Agro Morphologique de la feverole (Vicia faba L. var. minuta (Hort.ex Alef.) Mansf.) locale et perspectives d'amélioration génétique. Projet de fin d'Etudes. INAT, 88 p.

Dagnelie, P. (1965). A propos de quelques méthodes de comparaisons multiples de moyennes. Biom. Prascim., 6: II5-I24.

— (I986). Théorie et méthodes statistiques, Applications agronomiques. Les presses agronomiques de Gembloux, A.S.B.L. Belgique: vol.2, 463 p.

Duc, G., Bao,S., Baum, M., Redden, B., Sadiki, M., Jose Suso, M., Vishniakova, M. \& Zong, X. (20Io). Diversity maintenance and use of Vicia faba L. genetic resources. Field Crops Research II5, 270-278.

El Gazzah, M. \& Chalabi, N. (I995). Ressources génétiques et amélioration des plantes. In Quel avenir pour l'amélioration des plantes? Ed.AUPELF-UREF. John Libbey Eurotext. Paris, I23-I29.

Escadafal, R. (I985). Carte des ressources en sols de la Tunisie au 1/200 oooème, Feuille de Tataouine, République Tunisienne ; Ministère de l'Agriculture, Direction des Sols : $40 \mathrm{p}$.

Floret, C. \& Pontanier, R. (I982). L'aridité en Tunisie présaharienne: climat, sol, végétation et aménagement. Travaux et documents de l'OROSTOM N ${ }^{\circ}$ 50: $544 \mathrm{p}$.

Halila, M.H. (I995). Les ressources phylogénétiques des fabacées alimentaires en Tunisie : situation actuelle et perspectives. Rapport de consultation sur l'étude de faisabilité du projet FEM/PNUD/FAO sur la conservation et la valorisation des ressources génétiques des plantes au Maghreb I995, 34 p. 
Le Floc'h, E. (I986). Carte bioclimatique de la Tunisie. Sous la direction de M. Gounot, CNRS Montpellier, France.

Le Houérou, H.N. (I969). La carte phyto-écologique de la Tunisie centrale au 1/500.000. ORSTOM, Paris.

Lefort-Buson, M., Hebert, Y. \& Damerval, C. (I988). Les outils d'évaluation de la diversité génétique et phénotypique. Agronomie 8 (3): 173-178.

Loumerem, M. (1998). Inventory of some cultivated landraces threatened by genetic erosion in southern Tunisia. Plant genetic Resources newsletter, I998, nII3: 8-12.

Loumerem, M., Moussa, M. \& Bellachheb, C. (2004). La collecte et l'étude de la diversité génétique des éspèces cultivées aux aménagements hydrauliques dans les régions arides tunisiennes. Revue des Régions Arides - ISSN 0330-7956, Actes de Séminaire international, Aridoculture et Cultures Oasiennes, 22-25/II/2004.

Mzabi, H. (I988). La Tunisie du Sud-Est 'Géographie d'une région fragile marginale et dépendante'. Thèse de doctorat d'Etat en Sciences Humaines et Sociales, Université de Tunis, Faculté des Sciences Humaines et Sociales, Département de géographie: $444 \mathrm{p}$.

Pernès, J. (I984). Centres de ressources génétiques et formation des personnels de gestion. In Jean Pernès avec la collaboration de A. Charrier, D. Combes, J.L. Guillaumet, J.M. Leblanc, M. Lourd, E. Nguyen Van, Y. et G. Second (éds) Gestion des ressources génétiques des plantes. Tome II. Manuel. Diffuseur: Technique et documentation - LAVOISIER, II, Lavoisier 75384 Paris cedex o8, 295-345.

Perrino, P., Robertson, L.D. \& Solh, M.B. (I99I). Maintenance, evaluation and use offaba bean germplasm collections: problems and prospects. Options Méditerranéennes- Série seminaries- nº: 2I-3I.

Pistrick, K., Loumerem, M. \& Haddad, M. (I994). Field studies of plant genetic resources in South Tunisia. Plant Genetic Resources Newsletter 98:I3-I7.

Rholf, F.J. (I990). Numerical taxonomy and multivariate analysis system. Applied Biostatistics Inc. USA. $356 \mathrm{p}$.

Tanno, K. \& Willcox, G. (2006). The origins of cultivation of Cicer arietinum L. and Vicia faba L.: early finds from Tell el-Kerkh, north-west Syria, late Ioth millennium B.P. Veget. Hist. Archaeobot. 15, 197-204.

Thalji, T. \& Shalaldeh, G. (2006). Effect of planting date on faba bean (Vicia faba L.) nodulation and performance under semiarid conditions. World Journal of Agricultural Sciences, Vol.2 (4), 463-479.

UPOV (2000). Examen de la conformité de la Législation de la Tunisie avec l'acte de 1991 de la Convention UPOV. CONSEIL, Trente-quatrième session ordinaire Genève, 26 octobre 2000.

Yahia, Y. (2008). Variabilité morpho-phénologique des populations de Vicia faba L. cultivées dans les régions arides tunisiennes. Mastère en Génétique et Bio ressources. Faculté des Sciences de Tunis, 78 p. 